\title{
Differential regulation of neurexin at glutamatergic and GABAergic synapses
}

\section{Giulia Pregno ${ }^{1}$, Elena Frola ${ }^{1}$, Stefania Graziano ${ }^{2}$, Annarita Patrizi $^{3}$, Federico Bussolino ${ }^{2}$, Marco Arese ${ }^{2}$ and Marco Sassoè-Pognetto ${ }^{1 *}$}

1 Department of Neuroscience, University of Turin, Torino, Italy

${ }^{2}$ Department of Oncological Sciences and Institute for Cancer Research and Treatment, University of Turin, Candiolo, Italy

${ }^{3}$ F.M. Kirby Neurobiology Center, Harvard Medical School, Children's Hospital, Boston, MA, USA

Edited by:

Arianna Maffei, SUNY Stony

Brook, USA

Reviewed by:

Angel L. De Blas, University

of Connecticut, USA

Lisa Mapelli, University of

Pavia, Italy

*Correspondence:

Marco Sassoè-Pognetto,

Department of Neuroscience,

University of Turin, Corso Massimo

d'Azeglio 52, I-10126 Torino, Italy.

e-mail: marco.sassoe@unito.it
Neurexins (Nrxs) have emerged as potential determinants of synaptic specificity, but little is known about their localization at central synapses. Here we show that Nrxs have a remarkably selective localization at distinct types of glutamatergic synapses and we reveal an unexpected ontogenetic regulation of Nrx expression at GABAergic synapses. Our data indicate that synapses are specified by molecular interactions that involve both Nrx-dependent and Nrx-independent mechanisms. We propose that differences in the spatio-temporal profile of Nrx expression may contribute to specify the molecular identity of synapses.

Keywords: neurexin, neuroligin, $\mathrm{GABA}_{A}$ receptor, synaptic specificity, cerebellum

\section{INTRODUCTION}

The assembly, validation, and specificity of synapses are thought to depend on trans-synaptic interactions between cell-adhesion molecules (Waites et al., 2005; Shen and Scheiffele, 2010; Williams et al., 2010). Neurexins (Nrxs) are among the best characterized adhesion molecules that have been implicated in synapse formation and synaptic specificity (Ichtchenko et al., 1995; Craig and Kang, 2007). When expressed in non-neuronal cells, Nrxs induce synapse formation on co-cultured neurons, suggesting that they function in the initial assembly of synaptic connections (Graf et al., 2004; Nam and Chen, 2005). Deletion of $\alpha$-Nrxs in mice results in a lethal phenotype characterized by a massive impairment in $\mathrm{Ca}^{2+}$-channel function and neurotransmitter release (Missler et al., 2003; Zhang et al., 2005). In Drosophila, mutation of the single Nrx gene causes severe structural defects of the neuromuscular junction, and corresponding alterations in synaptic transmission (Li et al., 2007). These genetic data suggest that although Nrxs may not be strictly required for synaptogenesis, they are crucial for the proper assembly and functional maturation of synapses. Accordingly, human genetic studies have evidenced that mutations in Nrx genes are linked to several psychiatric disorders, including autism and schizophrenia (reviewed in Südhof, 2008; Betancur et al., 2009).

There are three Nrx genes in mammals, each encoding $\alpha$ and $\beta$ transcripts, that are further subject to alternative splicing resulting in potentially thousands of different isoforms (Ullrich et al., 1995). These molecules bind to multiple, structurally diverse postsynaptic partners. In addition to neuroligins, that were the first characterized Nrx-binding partners, Nrxs bind to a variety of other molecules, including leucine-rich repeat transmembrane proteins (LRRTMs), neurexophilins, dystroglycan, $\mathrm{GABA}_{\mathrm{A}}$ receptors $\left(\mathrm{GABA}_{\mathrm{A}} \mathrm{Rs}\right)$, and cerebellin-1 (Cbln1; for review, see Siddiqui and Craig, 2011). These interactions are regulated at the level of mRNA alternative splicing, generating a complex molecular "code" that may be important for synapse specification (Boucard et al., 2005; Chih et al., 2006; Uemura et al., 2010). In vertebrates, Nrxs are synthesized throughout the brain in all excitatory and inhibitory neurons (Ullrich et al., 1995). Therefore, it is generally assumed that they have a general role in synapse development and act as presynaptic hub molecules that mediate synapse maturation via selective interactions with different ligands (Südhof, 2008; Siddiqui and Craig, 2011). However, the distribution of endogenous Nrxs at distinct types of excitatory and inhibitory synapses is largely unknown, due to the lack of antibodies suitable for immunohistochemistry.

In this study, we used a pan-Nrx antiserum raised against a conserved intracellular region. Using the cerebellum as a model system, we show that Nrxs have a selective localization at distinct types of glutamatergic synapses. We also demonstrate that GABAergic interneurons regulate the synaptic expression of Nrxs during postnatal development. These differences in localization suggest that Nrxs have distinct roles in the development of glutamatergic and GABAergic synapses.

\section{RESULTS \\ NEUREXINS ARE PRESENT AT MOST BUT NOT ALL CEREBELLAR SYNAPSES}

We tested the rabbit pan-Nrx antiserum on cerebellar sections processed with different fixation techniques. We found that Nrx epitopes were sensitive to prolonged aldehyde fixation, and weak fixation protocols were therefore used to visualize Nrx immunoreactivity (see Materials and Methods). Nrx labeling was punctate, supporting synaptic localization (Figure 1). Moreover, double labeling with another pan-Nrx antibody raised in chicken 


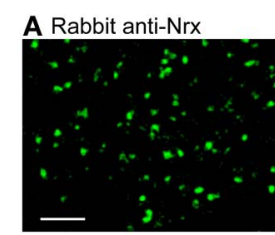

Chicken anti-Nrx
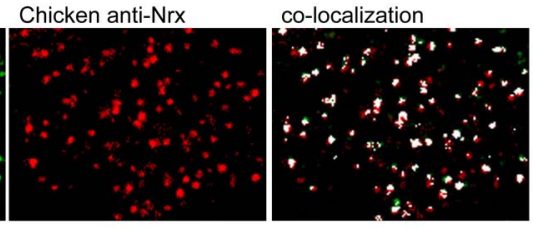

B $\operatorname{Nrx}(\mathrm{Rb})$
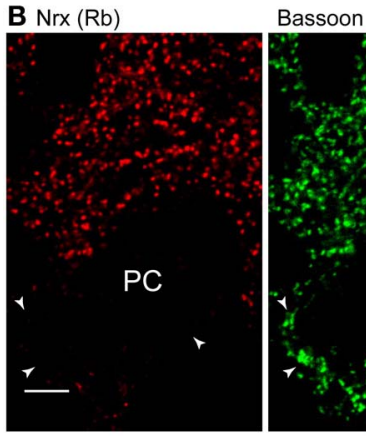

\section{Bassoon}

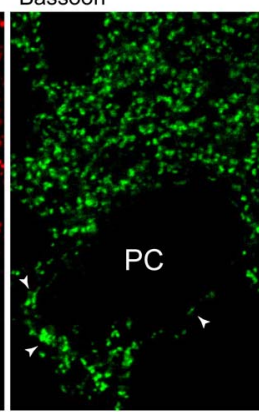

merge
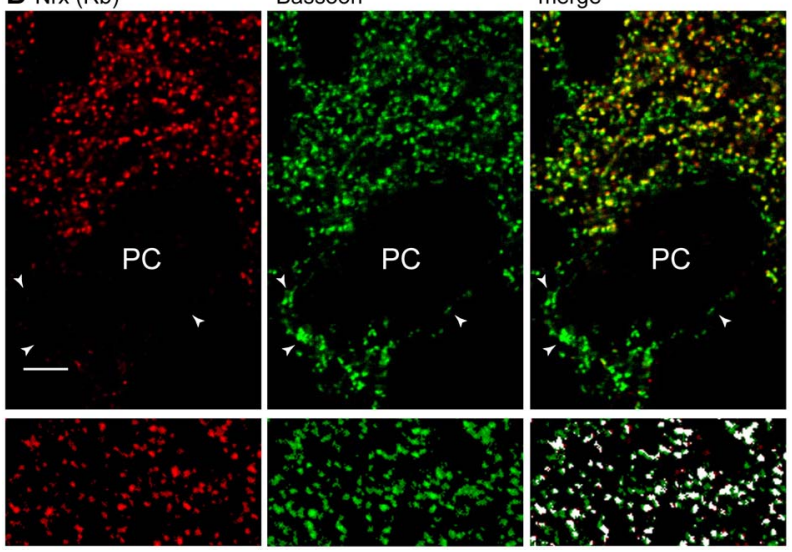

in the cerebellar

FIGURE 1 | Nrx localizes at a subset of synapses in the cerebellar
cortex. (A) Double labeling with pan-Nrx antibodies raised in rabbit (green) and chicken (red) resulted in substantial co-localization. All panels show segmented images that were processed with the Imaris co-localization algorithm (co-localized structures are shown in white). (B) Nrx antibodies produce punctate labeling that co-localizes extensively with the presynaptic marker bassoon. Note that bassoon-positive puncta (arrowheads) surrounding Purkinje cells (PC) are Nrx-negative. The lower panels show segmented images that were processed with the co-localization module. Scale bars: (A), $3 \mu \mathrm{m}$; (B), $5 \mu \mathrm{m}$.

(Dean et al., 2003) revealed extensive co-localization (Figure 1A), indicating that both antibodies recognize the same molecules. However, the rabbit antiserum produced a stronger labeling and was therefore used in all subsequent experiments.

To establish synaptic localization, we performed double immunofluorescence for Nrx and the presynaptic matrix protein bassoon (Figure 1B). Quantitative analysis of co-localization revealed that practically all Nrx-positive puncta in the molecular layer were associated with bassoon. However, Nrx was present in $\sim 80 \%$ of bassoon-positive synapses (4204 co-localized puncta out of a total of 5109 bassoon-positive puncta in two different cerebella), implying that some cerebellar synapses lack Nrx. In particular, perisomatic synapses surrounding PCs were Nrxnegative, suggesting that Nrx may be absent from GABA synapses (see below).

\section{NEUREXINS ARE PRESENT AT PF BUT NOT CF SYNAPSES}

The cerebellum provides an exquisite example of synaptic specificity. Purkinje cells (PCs), the principal neurons of the cerebellar cortex, receive glutamatergic input from two distinct sources: parallel fibers (PFs) establish synapses with spines located on the distal dendrites, whereas climbing fibers (CFs) contact spines located in the proximal dendritic domain (Yuste and Bonhoeffer, 2004; Cesa and Strata, 2009). Confocal imaging in L7-GFP mice showed that Nrx puncta were closely associated with PC spines, suggesting localization in PFs (Figure 2A). This was confirmed by immunogold labeling, showing gold particles at the presynaptic side of PF-PC synapses (Figure 2B). In contrast, we did not find Nrx signals in CF terminals, labeled with antibodies against the vesicular glutamate transporter VGluT2, indicating that in the cerebellar cortex Nrx is selectively localized at specific types of glutamatergic synapses (Figure 2C).

The presence of Nrxs at PF-PC synapses was further evidenced in double-labeling with the metabotropic glutamate receptor mGluRla, that labels selectively PC spines (Tanaka et al., 2000). These experiments revealed that Nrx puncta were clustered opposite to mGluRla-positive spines (Figure 2D). Notably, the pattern of Nrx expression in PF terminals was severely disrupted in mutant hotfoot mice, that lack the glutamate receptor GluRD2 (Mandolesi et al., 2009). In these animals, the size of Nrx puncta was significantly decreased (Kolmogorov-Smirnov, $p<0.001$, $n=3$ ) compared to the control situation (Figure 2F), as also documented by the presence of numerous small puncta (generally $<0.04 \mu \mathrm{m}^{2}$ ) characterized by considerably reduced brightness and fuzzy appearance (Figure 2E). These small puncta were generally not associated with PC spines (Figure 2E), suggesting that Nrx fails to form stable clusters when PFs are not connected to the appropriate postsynaptic targets. Moreover, the density of the larger Nrx puncta $\left(0.04-0.2 \mu \mathrm{m}^{2}\right)$ was significantly reduced in the hotfoot cerebellum (mean \pm SEM puncta/ $1000 \mu \mathrm{m}^{2}$ : control, $473.5 \pm 39$; hotfoot, $303 \pm 19 ; n=6$ sampling fields from 3 mice; unpaired $t$-test, $P=0.0061$ ), consistent with the robust reduction of synapses between PFs and PCs reported previously (Mandolesi et al., 2009). These data are in agreement with the idea that the integrity of PF synapses depends on a tripartite trans-synaptic complex comprising Nrx, the secreted glycoprotein Cbln1 and GluD2 (Matsuda et al., 2010; Uemura et al., 2010).

\section{NEUREXINS ARE MAINLY ASSOCIATED WITH DEVELOPING GABA SYNAPSES}

We next analyzed the expression of Nrxs at GABAergic synapses. In the mature cerebellum ( $>$ P30), Nrx was below detectable levels at inhibitory synapses labeled for the $\mathrm{GABA}_{\mathrm{A}} \mathrm{R} \alpha 1$ subunit (Figure 3A) or the scaffold molecule gephyrin (not shown). Quantitative evaluation of co-distribution in the molecular layer revealed that $\sim 3 \%$ of GABA synapses were Nrxpositive (162 co-distributed puncta out of a total of 5236 $\mathrm{GABA}_{\mathrm{A}} \mathrm{R}$-positive puncta). However, in postnatal mice (P5-P21) Nrx was found in practically all GABA synapses (co-distribution index at P15: 97\%, 2810 co-distributed puncta out of a total of $2901 \mathrm{GABA}_{\mathrm{A}} \mathrm{R}$-positive puncta), including perisomatic and axo-dendritic synapses made by basket cells and stellate cells (Figure 3B).

To validate these immunohistochemical findings, we isolated synaptosomes from cerebella of P15 and adult (>P30) mice, and we evaluated the potential of neuroligin-2 (NL2) to coimmunoprecipitate Nrx from developing and mature synapses. Because NL2 is present at GABA synapses since early stages of synaptogenesis (Patrizi et al., 2008), it can be effectively used to monitor developmental changes in the expression of interacting proteins. As expected, co-immunoprecipitation of NL2 from synaptosomes yielded considerably higher Nrx levels in juvenile 

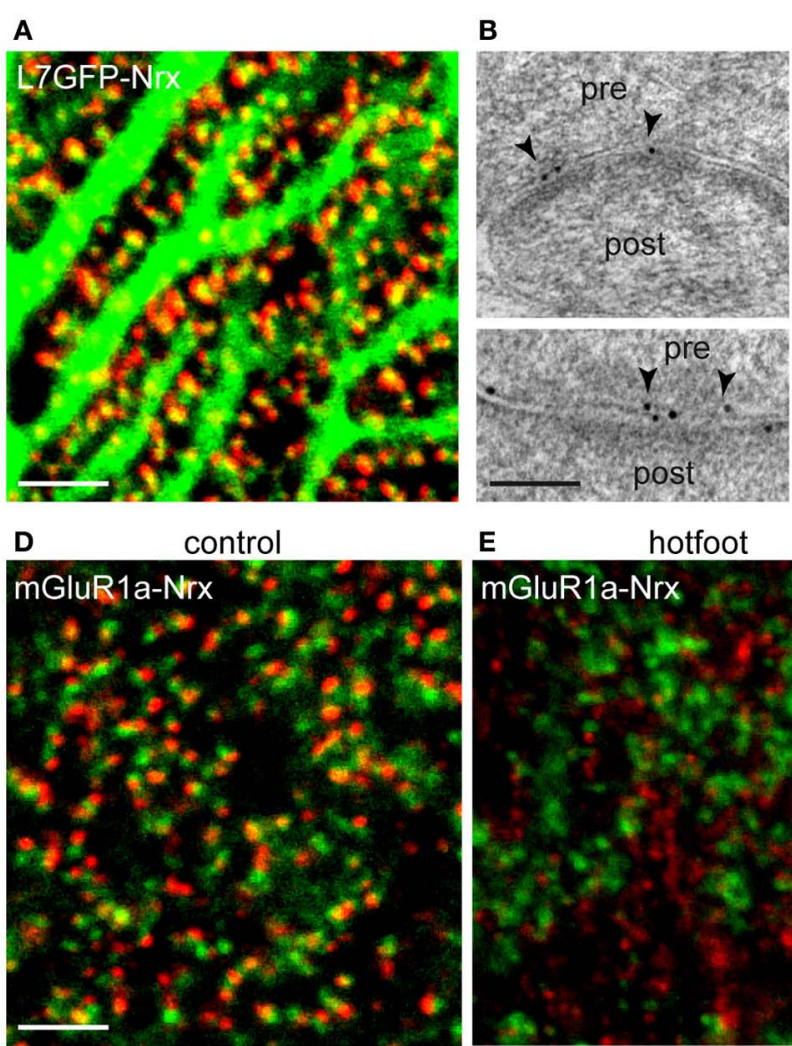

E

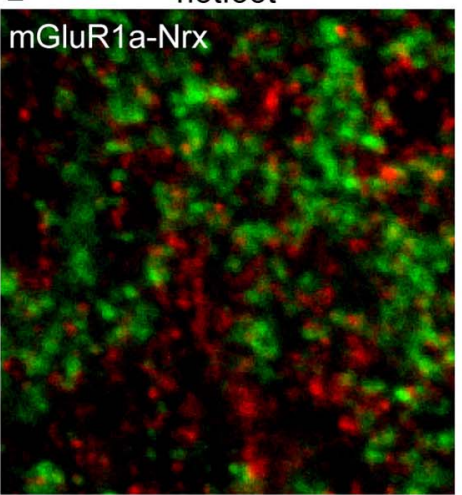

c
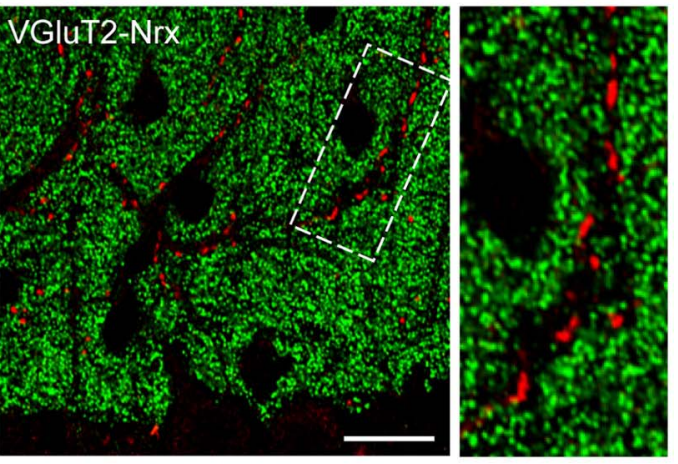

$\mathbf{F}$

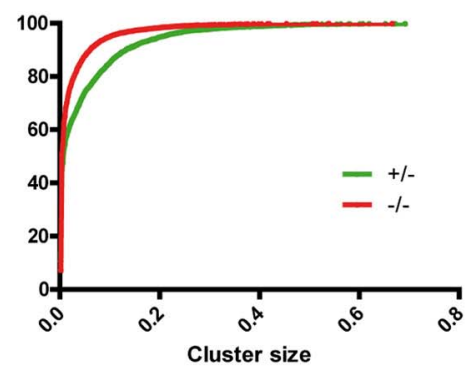

FIGURE 2 | Selective localization of Nrx at synapses made by PFs but not CFs. (A) Nrx puncta (red) are closely apposed to Purkinje cell spines in a L7-GFP mouse cerebellum. (B) Immunogold labeling (arrowheads) reveals the presence of $\mathrm{Nrx}$ at synapses between parallel fibers (pre) and Purkinje cell spines (post). (C) Nrx (green) is not associated with VGluT2-positive climbing fiber terminals (red). The boxed area is shown at higher magnification in the inset. (D,E) Double labeling for $\mathrm{Nrx}$ (red) and mGluR1a (green) in the cerebellum of control (D) and hotfoot (E) mice. Note the altered pattern of Nrx localization in the hotfoot mutant. (F) Cumulative plot showing the reduced size of Nrx puncta in hotfoot mice compared to control. Scale bars: $(\mathbf{A}, \mathbf{D}, \mathbf{E})$, $3 \mu \mathrm{m}$; (B), $100 \mathrm{~nm}$; (C), $18 \mu \mathrm{m}$. than in adult mice (Figure 3C), supporting the idea that Nrx is present in developing GABA synapses, but is downregulated at the end of the synaptogenic period.

We examined whether the expression of Nrx at developing GABA synapses depends on NL2 or $\mathrm{GABA}_{\mathrm{A}} \mathrm{Rs}$, that are Nrx-binding partners at inhibitory synapses (Graf et al., 2004; Varoqueaux et al., 2004; Zhang et al., 2010). We found no defects in synaptic localization of Nrx in P16 NL2 KO mice (Figure 3D; mean \pm SEM Nrx clusters $/ 50 \mu \mathrm{m}$ of perisomatic membrane: WT, $23.5 \pm 0.45, n=44$ cells; KO, $24.3 \pm 0.36, n=57$ cells; unpaired $t$-test, $p=0.1637)$. We then analyzed PC- $\Delta \alpha 1$ mice, that have a selective deletion of the $\mathrm{GABA}_{\mathrm{A}} \mathrm{R} \alpha 1$ subunit gene in PCs, resulting in complete loss of $\mathrm{GABA}_{\mathrm{A}}$ Rs (Briatore et al., 2010). Deletion of the $\alpha 1$ subunit is asynchronous among different PCs, resulting in a characteristic mosaic-like pattern, with $\alpha 1$-positive and $\alpha 1$-negative cells, in P14-P16 mice (Figure 3E). We found no differences in the synaptic localization of $\mathrm{Nrx}$ in $\alpha 1$-positive and $\alpha 1$ negative PCs of P16 PC- $\Delta \alpha 1$ mice (Figure 3E; mean \pm SEM Nrx clusters $/ 50 \mu \mathrm{m}$ of somatic membrane: $\alpha 1$-positive PCs, $19.9 \pm$ $0.64, n=33$ cells; $\alpha 1$-negative PCs, $19.6 \pm 0.46, n=32$ cells; unpaired $t$-test, $p=0.7365)$. These results suggest that neither NL2 nor GABA $A$ Rs are essential for Nrx localization at developing GABAergic synapses.
To establish whether the transient expression of $\mathrm{Nrx}$ is a general feature of GABA synapses, we analyzed co-distribution with the obligatory $\gamma 2$ subunit of synaptic $\mathrm{GABA}_{\mathrm{A}} \mathrm{Rs}$ in sensorimotor cortex of adult mice (Figure 3F). We found that in adult animals Nrx was associated with a small percentage of GABAergic synapses labeled with antibodies against the $\mathrm{GABA}_{\mathrm{A}} \mathrm{R}$ $\gamma 2$ subunit. Quantification in pyramidal neurons $(n=28$ cells from four mice) revealed that $\sim 30 \%$ of perisomatic synapses were Nrx-positive. Unfortunately, labeling for Nrx was generally quite challenging outside of the cerebellum, especially when combined with other antibodies, which precluded a detailed characterization of the Nrx-positive synapses. For the same reason, we could not analyze Nrx localization at developing synapses in postnatal mice. However, the limited co-distribution with $\mathrm{GABA}_{\mathrm{A}} \mathrm{Rs}$ substantiates the idea that Nrxs are scarcely represented at mature GABAergic synapses.

\section{DISCUSSION}

The three mammalian Nrx genes undergo extensive alternative splicing in their extracellular domain, potentially generating thousands of different isoforms (Ullrich et al., 1995). Nrxs are widespread in neurons, which has led to the general assumption that Nrx isoforms could determine synaptic properties 
A

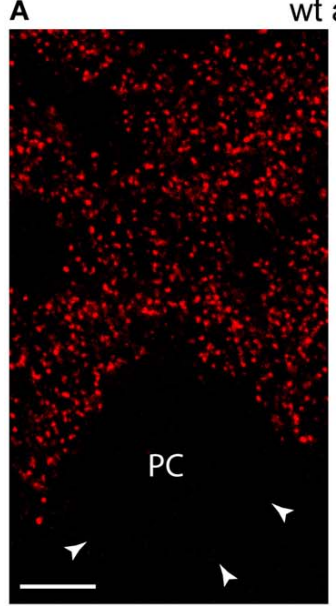

wt adult

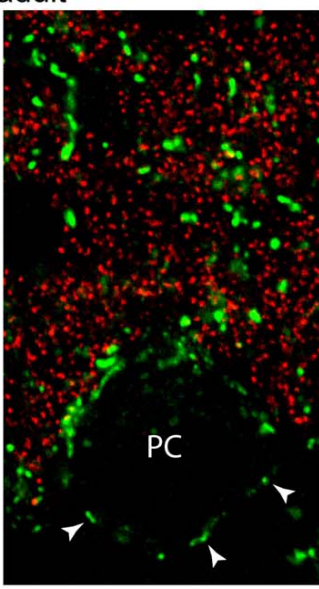

C

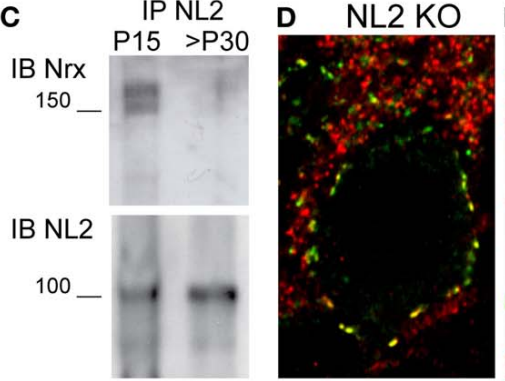

E

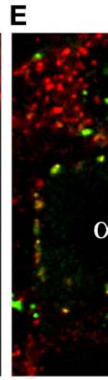

B

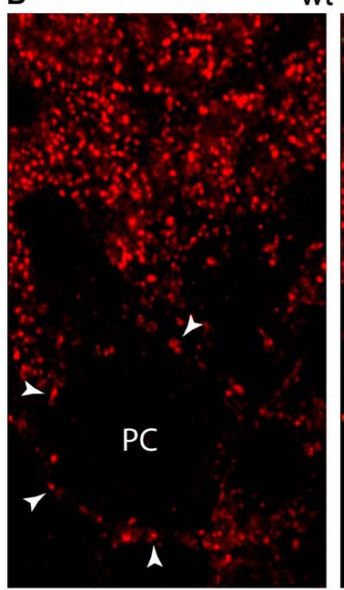

$\mathrm{PC} \Delta \alpha 1$

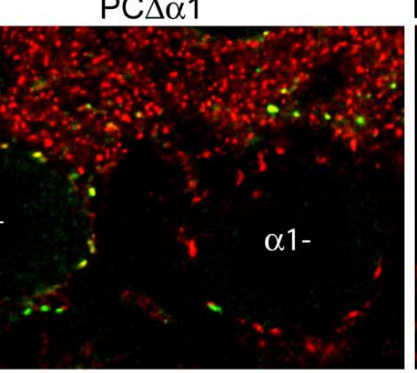

wt P15

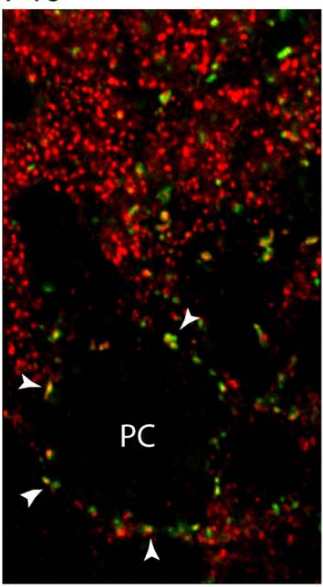

F wt adult - cortex

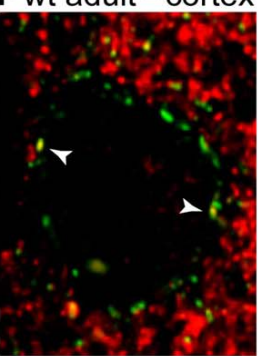

FIGURE 3 | Ontogenetic regulation of Nrxs at GABA synapses. All confocal images show double labeling for $\mathrm{Nrx}$ and $\mathrm{GABA}_{A} \mathrm{R} \alpha 1$ (A,B,D,E) or $\gamma 2$ (F). In all panels, labeling for $\mathrm{Nrx}$ is shown in red and labeling for $\mathrm{GABA}_{A} \mathrm{Rs}$ is shown in green. (A) Nrx is absent from GABA synapses in the cerebellum of an adult mouse. Arrowheads point to synaptic $G A B A_{A} R$ clusters outlining the cell body of a Purkinje cell (PC). (B) Nrx co-distributes with $G_{B A} A$

clusters in a P15 mouse cerebellum. (C) Higher amounts of Nrx are observed after co-immunoprecipitation with NL2 from synaptosomes obtained from P15 cerebella compared with >P30 cerebella. (D) Nrx persists at GABA synapses in a P16 NL2 KO mouse. (E) Nrx puncta outline the cell bodies of both $\mathrm{GABA}_{A} R$ a1-positive and $\mathrm{GABA}_{A} R$ a1-negative Purkinje cells in the cerebellum of a P16 PC- $\Delta \alpha 1$ mutant mouse. (F) Nrx is associated with only a few (arrowheads) $G_{A B A} R \gamma 2$-positive, perisomatic clusters in a cortical pyramidal neuron of an adult (>P30) mouse. Scale bar: (A,B,D-F), $6 \mu \mathrm{m}$. by interacting selectively with specific postsynaptic partners (Südhof, 2008; Williams et al., 2010; Siddiqui and Craig, 2011). Our present findings add a new dimension to the concept of a "molecular synaptic code" (Selimi et al., 2009), by showing that Nrxs undergo differential spatio-temporal regulation at distinct types of glutamatergic and GABAergic synapses.

We report two principal results. First, we show that Nrxs have a remarkably selective localization in PFs but not in CFs of the cerebellar cortex (Figures 2A-C). These afferents innervate distinct domains of the PC dendritic arbor, a process that depends on activity-dependent competition, as well as on differences in their molecular organization (Cesa and Strata, 2009; Kano and Hashimoto, 2009; Yuzaki, 2011). Our observations agree well with recent data showing that Nrx acts as a presynaptic ligand for the GluD2-Cbln1 complex at PF synapses (Matsuda et al., 2010; Uemura et al., 2010). Extensive evidence indicates that Cbln1, which is secreted by PFs, acts as a bi-directional receptor for both GluD2 and Nrx, thus providing a physical linkage between the postsynaptic density in PC spines and the active zone in PF terminals (Uemura et al., 2010; Yuzaki, 2011). Consistent with this idea, we found that Nrx clusters were altered in the cerebellum of hotfoot mice (Figure 2E), that lack GluD2. First, there was a significant reduction in the density of the larger Nrx puncta, that presumably corresponded to synapses between PFs and PCs. Second, many small clusters unrelated to PC spines became apparent in the molecular layer, suggesting that the presynaptic localization of Nrx depends on the structural integrity of PF synapses and proper molecular interactions mediated by the GluD2-Cbln1 complex. On a more general level, the selective localization of $\mathrm{Nrx}$ at PF but not CF synapses indicates that these glutamatergic contacts depend on trans-synaptic interactions mediated by distinct complexes of adhesion molecules. While the Nrx/Cbln1/GluD2 triad likely represents a "molecular code" for PF synapses (Uemura et al., 2010), the identity of adhesion molecules expressed at CF synapses remains to be determined. Synaptic protein profiling (Selimi et al., 2009) and other proteomic strategies may help to decipher signaling pathways that are selectively involved in the specification of these glutamatergic contacts.

Another important finding was that Nrxs undergo a developmentally regulated expression at GABA synapses. The situation in the cerebellar cortex is paradigmatic. Here Nrx was associated with GABA synapses during the entire period of postnatal development, but was strongly downregulated at the end of 
synaptogenesis, resulting in undetectable levels in mature circuits (Figures 3A,B). This transient expression is in perfect agreement with in vitro analyses, showing that overexpression of Nrxs in cultured neurons did not impair existing GABA synapses, but affected selectively the properties of developing contacts (Zhang et al., 2010). Together, these data suggest that Nrx may act to regulate the functional maturation of developing GABAergic synapses. Interestingly, a recent study in Caenorhabditis showed that Nrx and NL mediate a retrograde synaptic signal that inhibits neurotransmitter release by adjusting the rate and duration of synaptic vesicle release ( $\mathrm{Hu}$ et al., 2012). Studies on cultured neurons also have demonstrated that Nrxs suppress GABAergic synaptic transmission by direct binding to $\mathrm{GABA}_{\mathrm{A}}$ Rs (Zhang et al., 2010). Therefore, we speculate that the ontogenetically regulated expression of Nrx at GABA synapses may represent a mechanism to adjust the levels of transmission at a time when GABA has a strong influence over the developmental assembly of neuronal circuits (Akerman and Cline, 2007; Huang and Scheiffele, 2008; Wang and Kriegstein, 2009).

The expression profile in neocortex was quite different, as Nrx was retained in a subset of GABA synapses in adult mice (Figure 3F). It is of note that Nrxs bind directly to $\alpha 1-\mathrm{GABA}_{\mathrm{A}} \mathrm{Rs}$ (Zhang et al., 2010), that are the unique type of $G_{A B A} R$ in PCs and have a more limited distribution in neocortical neurons. It is possible that Nrxs may be differently regulated at distinct types of inhibitory synapses characterized by different $\mathrm{GABA}_{\mathrm{A}} \mathrm{R}$ subtypes. Unfortunately, we have been unable to address this question due to the difficulties in detecting endogenous Nrxs. It should also be mentioned that the pan-Nrx antiserum used in this study was raised against a C-terminal amino acid sequence of Nrx1 that is largely conserved in the Nrx2 and Nrx3 isoforms (see Materials and Methods). While immunoblot analyses have shown that the antiserum recognizes all Nrx isoforms (Bottos et al., 2009), we cannot exclude that our observations are biased by higher affinity of the antiserum for Nrxl than for the other Nrxs. The situation is also complicated by the fact that other than the original in situ hybridization mapping, that revealed differential but overlapping distribution of Nrx isoforms in distinct classes of neurons (Ullrich et al., 1995), very little has become available about the expression of the different isoforms and their regulation at the cellular and synaptic levels.

Our current results suggest that the localization of $\operatorname{Nrx}$ at developing GABA synapses is not influenced by binding to its postsynaptic ligands NL2 and $\mathrm{GABA}_{\mathrm{A}}$ Rs (Figures 3D,E). However, our observations in NL2 KO mice may be confounded by compensatory changes involving other NL isoforms. Indeed, we have observed a strong upregulation of NL3 and NL4 in PCs of NL2 KO mice (data not shown). Moreover, cellular imaging after artificial expression of tagged Nrx isoforms in cortical interneurons demonstrated that the presynaptic localization of $\operatorname{Nrx} 1 \beta$ depends on binding to postsynaptic ligands ( $\mathrm{Fu}$ and Huang, 2010). On the other hand, no $\mathrm{GABA}_{\mathrm{A}}$ Rs are present in PCs of PC- $\Delta \alpha 1$ mice (Briatore et al., 2010), suggesting that binding to $\mathrm{GABA}_{\mathrm{A}} \mathrm{Rs}$ is not essential for presynaptic stabilization of Nrxs.

While there is no doubt that endogeneous Nrxs are presynaptic, it has been proposed that these adhesion molecules may partly localize postsynaptically (Taniguchi et al., 2007). Unfortunately, postembedding electron microscopy yielded relatively weak labeling, precluding quantitative analysis of gold particle distribution at PF-PC synapses. However, the precise apposition of NRXpositive puncta with $\mathrm{PC}$ spines evidenced by confocal microscopy (Figure 2D) is consistent with a predominantly presynaptic localization.

In conclusion, our findings suggest that synapses are specified by molecular interactions that involve both Nrx-dependent and Nrx-independent mechanisms. Moreover, the complex spatio-temporal expression profile reported here suggests that ontogenetic regulation of Nrxs is of crucial importance for determining how synapses form and acquire their functional maturation.

\section{MATERIALS AND METHODS MICE}

Most of the experiments described in this study were performed on WT mice of the C57BL/6 strain and transgenic mice expressing GFP selectively in PCs (L7-GFP mice). In addition, three lines of mutant mice were used. Hotfoot mice are spontaneous mutants with a deletion of the glutamate receptor GluRD2 (Lalouette et al., 1998). GluD2 is expressed selectively in PC spines innervated by PFs and is crucial for the formation and maintenance of PF synapses (Kashiwabuchi et al., 1995; Yuzaki, 2003). Like GluRD2 knockout (KO) mice, hotfoot mutants have a reduced number of synapses between PFs and PCs (Mandolesi et al., 2009). The generation of PC- $\Delta \alpha 1$ mice has been described elsewhere (Briatore et al., 2010). Deletion of the $\mathrm{GABA}_{\mathrm{A}} \mathrm{R} \alpha 1$ subunit gene was driven by Cre recombinase expressed under control of the $\mathrm{L} 7$ promoter, which resulted in a characteristic mosaic pattern, with $\alpha 1$-positive and $\alpha 1$-negative cells, in P14-P16 mice (Figure 3E). Finally, NL2 KO mice (Varoqueaux et al., 2006) were kindly provided by Nils Brose (Max-Planck-Institute of Experimental Medicine and Center for Molecular Physiology of the Brain, Göttingen, Germany).

The experimental procedures were designed in accordance with national (Legislative Decree 116/92 and law n. 413/1993) and international (Directive 86/609/EEC and the recommendation 2007/526/EC from the European Community) laws and policies, and approved by the Italian Ministry of Health (Department of Public Veterinary Health) and by the ethical committee of Turin University.

\section{ANTIBODIES}

We used an affinity-purified, pan-Nrx antiserum produced in rabbits as described in detail elsewhere (Bottos et al., 2009). This antibody was raised against the peptide AKSANKNKKNKDKEYYV located in the intracellular region of the $\alpha$ and $\beta$ isoforms of mouse Nrxl. This region has high homology to Nrx2 (PKTPSKAKKNKDKEYYV) and Nrx3 (SKSGHKKQKNKDKEYYV). Consistent with this, the antiserum recognized all Nrx isoforms in immunoblotting of transfected HeLa cells. Another antiserum (kindly provided by Peter Scheiffele, Biozentrum, Basel, Switzerland) was raised in chicken against a recombinant neurexin-GST fusion protein containing the cytoplasmic tail of Nrxl (Dean et al., 2003). 
This antiserum recognized two bands with apparent molecular weights corresponding to $\alpha$ and $\beta$ Nrxs in western blots of total cerebellar lysates. A rabbit antiserum against NL2 was obtained from Synaptic Systems (cat. no. 129203). Guinea pig antisera against the vesicular glutamate transporter VGluT2 (Chemicon, $\mathrm{AB} 2251$ ) and the metabotropic glutamate receptor mGluR1a (kindly provided by Masahiko Watanabe, Hokkaido University, Sapporo, Japan) were used to label respectively CF terminals and PC spines (Tanaka et al., 2000; Fremeau et al., 2001). GABA synapses were identified with guinea pig antibodies against the $\alpha 1$ and $\gamma 2$ subunits of GABA $_{\mathrm{A}}$ Rs (kindly provided by Jean-Marc Fritschy, University of Zurich, Switzerland). The monoclonal antibody against bassoon was obtained from Stressgen (cat. no. VAM-PS003).

\section{IMMUNOFLUORESCENCE AND CONFOCAL MICROSCOPY}

Detection of Nrx required a brief fixation protocol described in detail elsewhere (protocol B in Schneider Gasser et al., 2006; see also Patrizi et al., 2008). In some cases, mice were perfused with $2 \%$ formaldehyde, and the brains were postfixed in the same fixative overnight. This fixation protocol allowed simultaneous detection of Nrx and VGluT2. Confocal images were acquired with a laser scanning confocal microscope (Zeiss LSM5 Pascal) using the multitrack mode to avoid fluorescence crosstalk. Synaptic puncta were analyzed on images acquired with a $\times 100$ oil-immersion objective (1.4 NA) at a magnification of $8.1 \times$ $10^{-3} \mu \mathrm{m}^{2} /$ pixel, and the pinhole set at 1 Airy unit. The images were processed with the image-analysis program Imaris (release 4.2; Bitplane). Clusters were quantified on segmented images with NIH Image J software. Co-localization was estimated on segmented images with the Imaris co-localization module (see Viltono et al., 2008). The density of perisomatic clusters was determined by counting manually Nrx puncta surrounding the cell body of PCs.

\section{IMMUNOGOLD LABELING}

Juvenile (P17) mice $(n=2)$ were perfused with $2 \%$ formaldehyde and $0.1 \%$ glutaraldehyde in sodium acetate buffer $(\mathrm{pH} 6)$ for $2 \mathrm{~min}$, followed by $1 \mathrm{~h}$ perfusion with $2 \%$ formaldehyde and $0.1 \%$ glutaraldehyde in $0.1 \mathrm{M}$ borate buffer ( $\mathrm{pH} 9$ ). Brains were postfixed in the second fixative solution overnight. Tissue blocks from the cerebellar vermis were freeze-substituted and embedded in Lowicryl HM20. Ultrathin sections were processed for the immunogold method using as secondary antibodies goat Fab fragments coupled to $10 \mathrm{~nm}$ colloidal gold particles (SassoèPognetto and Ottersen, 2000).

\section{REFERENCES}

Akerman, C. J., and Cline, H. T. (2007). Refining the roles of GABAergic signaling during neural circuit formation. Trends Neurosci. 30, 382-389.

Betancur, C., Sakurai, T., and Buxbaum, J. D. (2009). The emerging role of synaptic cell-adhesion pathways in the pathogenesis of autism spectrum disorders. Trends Neurosci. 32, 402-412.

\section{SYNAPTOSOMAL PREPARATION AND CO-IMMUNOPRECIPITATION}

Synaptosome extraction was performed using the Syn-PER Reagent (Thermo Scientific) following the manufacturer's protocol. Frozen cerebella obtained from P15 and adult (>P30) mice were disgregated with a tissue potter and lysed on ice with Syn-PER Reagent ( $10 \mathrm{ml}$ per gram of tissue) added with protease and phosphatase inhibitors $(50 \mu \mathrm{g} / \mathrm{mL}$ pepstatin, $50 \mu \mathrm{g} / \mathrm{mL}$ leupeptin, $10 \mu \mathrm{g} / \mathrm{mL}$ aprotinin, $1 \mathrm{mM} \mathrm{PMSF}, 100 \mu \mathrm{M}$ $\mathrm{ZnCl}_{2}, 1 \mathrm{mM} \mathrm{Na}$ orthovanadate, and $10 \mathrm{mM} \mathrm{NaF}$ ). The solution was centrifuged at $1200 \mathrm{~g}$ for $10 \mathrm{~min}$ at $4^{\circ} \mathrm{C}$, the pellet was discarded and the remaining supernatant was centrifuged at $10,000 \mathrm{~g}$ for $45 \mathrm{~min}$ at $4^{\circ} \mathrm{C}$. The resulting pellet was resuspended in $2 \mathrm{ml}$ of lysis buffer $[10 \mathrm{mM}$ Tris $\mathrm{HCl}, \mathrm{pH} 7.5$; $150 \mathrm{mM} \mathrm{NaCl} ; 5 \mathrm{mM}$ EDTA, pH 8; 10\% glycerol; 1\% Triton $\mathrm{X}-100$; and 1\% 3-[(3-Cholamidopropyl)dimethylammonio]1propanesulfonate (CHAPS)] with protease and phosphatase inhibitors $(50 \mu \mathrm{g} / \mathrm{mL}$ pepstatin, $50 \mu \mathrm{g} / \mathrm{mL}$ leupeptin, $10 \mu \mathrm{g} / \mathrm{mL}$ aprotinin, $1 \mathrm{mM}$ PMSF, $100 \mu \mathrm{M} \mathrm{ZnCl}, 1 \mathrm{mM} \mathrm{Na}$ orthovanadate, and $10 \mathrm{mM} \mathrm{NaF}$ ). After quantification with the BCA Protein Assay Reagent Kit (Pierce Chemical Co.), samples (5 mg of total proteins) were precleared with protein A-Sepharose (Amersham Biosciences) and incubated $1 \mathrm{~h}$ with the rabbit anti-Nrx antibody $(1.5 \mu \mathrm{g} / \mathrm{mg})$ or the rabbit anti-NL2 antibody $(2.5 \mu \mathrm{g} / \mathrm{mg})$. Immune complexes were recovered on protein A-Sepharose overnight and washed 4 times. Proteins were separated by $4-15 \%$ SDS/PAGE electrophoresis gel, transferred to polyvinylidene difluoride membrane (Millipore), and detected by immunoblot. Immunoreactive proteins were identified with an HRP-conjugated secondary antibody (Jackson ImmunoResearch) and visualized by an ECL system (Amersham Biosciences).

\section{ACKNOWLEDGMENTS}

We thank Nils Brose and Frédérique Varoqueaux (Göttingen) for providing NL2 KO mice, Piergiorgio Strata and Ferdinando Rossi (Torino) for the L7-GFP and hotfoot mice, Jean-Marc Fritschy (Zurich), Masahiko Watanabe (Sapporo) and Peter Scheiffele (Basel) for providing antibodies. This study was supported by Compagnia di San Paolo (grant 2007 and progetti di Ricerca di Ateneo 2011 to Marco Sassoè-Pognetto), the Italian MIUR (Prin 2008KN7J7J to Marco Sassoè-Pognetto), Association Française contre les Myopathies (AFM ref. n. 15299 to Marco Sassoè-Pognetto), Italian Association for Cancer Research (AIRC; IG11503 to Marco Arese, IG10133 to Federico Bussolino) and Italian MIUR (FIRB, contract: RBAP11BYNP, project: Newton). Giulia Pregno is supported by a fellowship from Fondazione CRT (Progetto Lagrange).

for trans-synaptic cell adhesion mediated by binding of neuroligin 1 to alpha- and beta-neurexins. Neuron 48, 229-236.

Briatore, F., Patrizi, A., Viltono, L., Sassoè-Pognetto, M., and Wulff, P. (2010). Quantitative organization of GABAergic synapses in the molecular layer of the mouse cerebellar cortex. PLoS ONE 5:e12119. doi: 10.1371/journal.pone.0012119
Cesa, R., and Strata, P. (2009). Axonal competition in the synaptic wiring of the cerebellar cortex during development and in the mature cerebellum. Neuroscience 162, 624-632. tem and contribute to its functions. Proc. Natl. Acad. Sci. U.S.A. 106, 20782-20787.

Boucard, A. A., Chubykin, A. A., Comoletti, D., Taylor, P., and Südhof, T. C. (2005). A splice code
Chih, B., Gollan, L., and Scheiffele, P. (2006). Alternative splicing controls selective trans-synaptic interactions of the neuroligin-neurexin complex. Neuron 51, 171-178. 
Craig, A. M., and Kang, Y. (2007). Neurexin-neuroligin signaling in synapse development. Curr. Opin. Neurobiol. 17, 43-52.

Dean, C., Scholl, F. G., Choih, J., DeMaria, S., Berger, J., Isacoff, E., et al. (2003). Neurexin mediates the assembly of presynaptic terminals. Nat. Neurosci. 6, 708-716.

Fremeau, R. T. Jr., Troyer, M. D., Pahner, I., Nygaard, G. O., Tran, C. H., Reimer, R. J., et al. (2001). The expression of vesicular glutamate transporters defines two classes of excitatory synapse. Neuron 31, 247-260.

Fu, Y., and Huang, Z. J. (2010). Differential dynamics and activitydependent regulation of alphaand beta-neurexins at developing GABAergic synapses. Proc. Natl. Acad. Sci. U.S.A. 107, 22699-22704.

Graf, E. R., Zhang, X., Jin, S. X., Linhoff, M. W., and Craig, A. M. (2004). Neurexins induce differentiation of GABA and glutamate postsynaptic specializations via neuroligins. Cell 119, 1013-1026.

Hu, Z., Hom, S., Kudze, T., Tong, X. J., Choi, S., Aramuni, G., et al. (2012). Neurexin and neuroligin mediate retrograde synaptic inhibition in $C$. elegans. Science 337, 980-984.

Huang, Z. J., and Scheiffele, P. (2008). GABA and neuroligin signaling: linking synaptic activity and adhesion in inhibitory synapse development. Curr. Opin. Neurobiol. 18, 77-83.

Ichtchenko, K., Hata, Y., Nguyen, T., Ullrich, B., Missler, M., Moomaw, C., et al. (1995). Neuroligin 1: a splice site-specific ligand for betaneurexins. Cell 81, 435-443.

Kano, M., and Hashimoto, K. (2009). Synapse elimination in the central nervous system. Curr. Opin. Neurobiol. 19, 154-161.

Kashiwabuchi, N., Ikeda, K., Araki, K., Hirano, T., Shibuki, K., Takayama, C., et al. (1995). Impairment of motor coordination, Purkinje cell synapse formation, cerebellar longterm depression in GluR delta 2 mutant mice. Cell 81, 245-252.

Lalouette, A., Guénet, J. L., and Vriz, S. (1998). Hotfoot mouse mutations affect the delta 2 glutamate receptor gene and are allelic to lurcher. Genomics 50, 9-13.

Li, J., Ashley, J., Budnik, V., and Bhat, M. A. (2007). Crucial role of Drosophila neurexin in proper active zone apposition to postsynaptic densities, synaptic growth, and synaptic transmission. Neuron 55, 741-755.

Mandolesi, G., Autuori, E., Cesa, R., Premoselli, F., Cesare, P., and Strata, P. (2009). GluRdelta2 expression in the mature cerebellum of hotfoot mice promotes parallel fiber synaptogenesis and axonal competition. PLOS ONE 4:e5243. doi: 10.1371/journal.pone.0005243

Matsuda, K., Miura, E., Miyazaki, T., Kakegawa, W., Emi, K., Narumi, S., et al. (2010). Cbln1 is a ligand for an orphan glutamate receptor delta2, a bidirectional synapse organizer. Science 328, 363-368.

Missler, M., Zhang, W., Rohlmann, A., Kattenstroth, G., Hammer, R. E., Gottmann, K., et al. (2003). Alphaneurexins couple $\mathrm{Ca} 2+$ channels to synaptic vesicle exocytosis. Nature 423, 939-948.

Nam, C. I., and Chen, L. (2005). Postsynaptic assembly induced by neurexin-neuroligin interaction and neurotransmitter. Proc. Natl. Acad. Sci. U.S.A. 102, 6137-6142.

Patrizi, A., Scelfo, B., Viltono, L., Briatore, F., Fukaya, M., Watanabe, M., et al. (2008). Synapse formation and clustering of neuroligin-2 in the absence of GABAA receptors. Proc. Natl. Acad. Sci. U.S.A. 105, 13151-13156.

Sassoè-Pognetto, M., and Ottersen, O. P. (2000). Organization of ionotropic glutamate receptors at dendrodendritic synapses in the rat olfactory bulb. J. Neurosci. 20, 2192-2201.

Schneider Gasser, E. M., Straub, C. J., Panzanelli, P., Weinmann, O., Sassoè-Pognetto, M., and Fritschy, J. M. (2006). Immunofluorescence in brain sections: simultaneous detection of presynaptic and postsynaptic proteins in identified neurons. Nat. Protoc. 1, 1887-1897.

Selimi, F., Cristea, I. M., Heller, E., Chait, B. T., and Heintz, N. (2009). Proteomic studies of a single CNS synapse type: the parallel fiber/purkinje cell synapse. PLoS Biol. 7:e83. doi: 10.1371/journal.pbio. 1000083

Shen, K., and Scheiffele, P. (2010). Genetics and cell biology of building specific synaptic connectivity. Annu. Rev. Neurosci. 33, 473-507.
Siddiqui, T. J., and Craig, A. M. (2011). Synaptic organizing complexes. Curr. Opin. Neurobiol. 21, 132-143.

Südhof, T. C. (2008). Neuroligins and neurexins link synaptic function to cognitive disease. Nature 455, 903-911.

Tanaka, J., Nakagawa, S., Kushiya, E., Yamasaki, M., Fukaya, M., Iwanaga, T., et al. (2000). Gq protein alpha subunits Galphaq and Galphal1 are localized at postsynaptic extrajunctional membrane of cerebellar Purkinje cells and hippocampal pyramidal cells. Eur. J. Neurosci. 12, 781-792.

Taniguchi, H., Gollan, L., Scholl, F. G., Mahadomrongkul, V., Dobler, E., Limthong, N., et al. (2007). Silencing of neuroligin function by postsynaptic neurexins. J. Neurosci. 27, 2815-2824.

Uemura, T., Lee, S. J., Yasumura, M., Takeuchi, T., Yoshida, T., Ra, M., et al. (2010). Trans-synaptic interaction of GluRdelta2 and Neurexin through Cbln1 mediates synapse formation in the cerebellum. Cell 141, 1068-1079.

Ullrich, B., Ushkaryov, Y. A., and Südhof, T. C. (1995). Cartography of neurexins: more than 1000 isoforms generated by alternative splicing and expressed in distinct subsets of neurons. Neuron 14, 497-507.

Varoqueaux, F., Aramuni, G., Rawson, R. L., Mohrmann, R., Missler, M., Gottmann, K., et al. (2006). Neuroligins determine synapse maturation and function. Neuron 51, 741-754.

Varoqueaux, F., Jamain, S., and Brose, N. (2004). Neuroligin 2 is exclusively localized to inhibitory synapses. Eur. J. Cell Biol. 83, 449-456.

Viltono, L., Patrizi, A., Fritschy, J. M., and Sassoè-Pognetto, M. (2008) Synaptogenesis in the cerebellar cortex: differential regulation of gephyrin and GABAA receptors at somatic and dendritic synapses of Purkinje cells. J. Comp. Neurol. 508, 579-591.

Waites, C. L., Craig, A. M., and Garner, C. C. (2005). Mechanisms of vertebrate synaptogenesis. Annu. Rev. Neurosci. 28, 251-274.

Wang, D. D., and Kriegstein, A. R. (2009). Defining the role of GABA in cortical development. J. Physiol. 587, 1873-1879.

Williams, M. E., de Wit, J., and Ghosh, A. (2010). Molecular mechanisms of synaptic specificity in developing neural circuits. Neuron 68, 9-18.

Yuste, R., and Bonhoeffer, T. (2004). Genesis of dendritic spines: insights from ultrastructural and imaging studies. Nat. Rev. Neurosci. 5, 24-34.

Yuzaki, M. (2003). The delta2 glutamate receptor: 10 years later. Neurosci. Res. 46, 11-22.

Yuzaki, M. (2011). Cbln1 and its family proteins in synapse formation and maintenance. Curr. Opin. Neurobiol. 21, 215-220.

Zhang, C., Atasoy, D., Araç, D., Yang, X., Fucillo, M. V., Robison, A. J., et al. (2010). Neurexins physically and functionally interact with GABA(A) receptors. Neuron 66, 403-416.

Zhang, W., Rohlmann, A., Sargsyan, V., Aramuni, G., Hammer, R. E., Südhof, T. C., et al. (2005). Extracellular domains of alphaneurexins participate in regulating synaptic transmission by selectively affecting $\mathrm{N}$ - and $\mathrm{P} / \mathrm{Q}-$ type Ca2+ channels. J. Neurosci. 25, 4330-4342.

Conflict of Interest Statement: The authors declare that the research was conducted in the absence of any commercial or financial relationships that could be construed as a potential conflict of interest.

Received: 03 December 2012; accepted: 18 March 2013; published online: 04 April 2013.

Citation: Pregno G, Frola E, Graziano S, Patrizi A, Bussolino F, Arese $M$ and Sassoè-Pognetto M (2013) Differential regulation of neurexin at glutamatergic and GABAergic synapses. Front. Cell. Neurosci. 7:35. doi: 10.3389/fncel. 2013.00035

Copyright (C) 2013 Pregno, Frola, Graziano, Patrizi, Bussolino, Arese and Sassoè-Pognetto. This is an open-access article distributed under the terms of the Creative Commons Attribution License, which permits use, distribution and reproduction in other forums, provided the original authors and source are credited and subject to any copyright notices concerning any third-party graphics etc. 